\title{
Narrativa
}

Nuova serie

$41 \mid 2019$

Narrativa italiana degli anni Duemila: cartografie e percorsi

\section{La biofiction italiana iper-contemporanea}

Marco Mongelli

\section{(2) OpenEdition}

\section{Journals}

\section{Edizione digitale}

URL: https://journals.openedition.org/narrativa/360

DOI: $10.4000 /$ narrativa.360

ISSN: 2804-1224

\section{Editore}

Presses universitaires de Paris Nanterre

\section{Edizione cartacea}

Data di pubblicazione: 1 décembre 2019

Paginazione: 105-113

ISBN: 978-2-84016-350-3

ISSN: $1166-3243$

\section{Notizia bibliografica digitale}

Marco Mongelli, «La biofiction italiana iper-contemporanea», Narrativa [Online], 41 | 2019, online dal 01 novembre 2021, consultato il 08 décembre 2021. URL: http://journals.openedition.org/narrativa/360 : DOI: https://doi.org/10.4000/narrativa.360

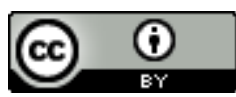

Narrativa est mise à disposition selon les termes de la Licence Creative Commons Attribution 4.0 International. 


\section{La biofiction italiana iper-contemporanea}

\section{RIASSUNTO}

Questo articolo intende fornire una breve disamina di un fenomeno letterario e transnazionale sempre più importante nella prosa italiana contemporanea: la biografia di finzione, o biofiction. La pervasività editoriale, e di immaginario, delle scritture biografiche a carattere letterario e/o finzionale degli ultimi tre decenni merita di essere indagata da diverse prospettive: questo contributo si concentrerà sul carattere specificamente letterario della biofiction.

\section{RÉSUMÉ}

Cet article se propose d'examiner un phénomène littéraire et transnational de plus en plus important dans la littérature italienne contemporaine: la biographie fictionnelle, ou biofiction. La prégnance sur le plan éditorial, et l'impact sur l'imaginaire, des écritures biographiques à caractère littéraire et / ou fictionnel de ces trois dernières décennies méritent d'être explorés sous différents angles: cette contribution porte sur le caractère spécifiquement littéraire de la biofiction.

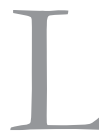

'interesse accordato dalla cultura contemporanea occidentale alla vita di una persona realmente esistita è eclatante e non si limita alla letteratura: il cinema, il teatro, il fumetto, la fotografia hanno acquisito negli ultimi tre decenni una dimensione biografica inedita e non estemporanea. Limitando lo sguardo alla letteratura, i titoli dei libri e delle collane editoriali degli ultimi anni testimoniano la pervasività di questa tendenza. Questa nuova fortuna della biografia riguarda due aspetti opposti della produzione culturale, rappresentando allo stesso tempo un fenomeno editoriale di massa e uno di ricerca letteraria: da un lato soddisfa il sentimento di conoscere le vite reali di personaggi più o meno famosi (e in un certo modo “illustri”); dall'altro rivela un'intenzione poetica precisa e consapevole da parte di molti autori contemporanei. Se il 
primo aspetto riguarda l'enorme produzione di biografie destinate soprattutto al grande pubblico, il secondo manifesta un interesse particolare e un'ambizione nuova. In effetti, è evidente che nel corso degli ultimi trent'anni la ricerca poetica di numerosi artisti si è orientata in questa direzione e ha prodotto delle opere importanti, spesso innovatrici nella forma e notevoli da un punto di vista estetico. Le due tipologie di produzione biografica conoscono un importante successo editoriale sotto le forme più diverse (letterarie, saggistiche o giornalistiche) e hanno come protagonisti scienziati, artisti, politici, sportivi, personalità dello spettacolo etc. Apprezzati da un pubblico molto diversificato, questi prodotti possono essere banalmente commerciali o al contrario estremamente letterari.

Solo in Italia i libri di natura letteraria a carattere genericamente biografico pubblicati negli ultimi due anni sono almeno una ventina: dalle voci manualistiche della Letteratura latina inesistente di Stefano Tonietto ${ }^{1}$ fino all'ultimo faction di Andrea Tarabbia, Madrigale senza suono ${ }^{2}$, passando per i testi romanzeschi di Montanaro $^{3}$, Janeczek ${ }^{4}$, Busato ${ }^{5}$, Franzosini ${ }^{6}$, Attadio ${ }^{7}$, Rovelli $^{8}$ e Cavezzali', per i racconti di Orecchio ${ }^{10}$, Baroncelli ${ }^{11}$, De Stefano ${ }^{12}$, Accatino ${ }^{13}$, Buonanno ${ }^{14}$ e Codignola ${ }^{15}$, o per delle biografie più schiettamente saggistiche come quelle di Petrignani e Santagata ${ }^{16}$.

1. Uscito nel 2017 per Quodlibet. Si tratta di una parodia del manuale scolastico in cui gli autori inclusi sono oggetto di rivisitazione immaginaria e finzionale.

2. Uscito il 21 febbraio del 2019 per Bollati Boringhieri.

3. Montanaro Giovanni, Guardami negli occhi, Milano, Feltrinelli, 2017.

4. Janeczek Helena, La ragazza con la Leica, Milano, Guanda, 2017.

5. Busato Nadia, Non sarò mai la brava moglie di nessuno, Milano, SEM, 2018.

6. Franzosini Edgardo, Rimbaud e la vedova, Milano, Skira, 2018.

7. AtтAdio Nicola, Dove nasce il vento. Vita di Nellie Bly, a free American girl, Milano, Bompiani, 2018.

8. Rovelli Marco, Il tempo delle ciliegie, Milano, Elèuthera, 2018.

9. Cavezzali Matteo, Icarus. Ascesa e caduta di Raul Gardini, Roma, minimumfax, 2018.

10. Orecchio Davide, Mio padre la rivoluzione, Roma, minimumfax, 2017. Anche se in realtà solo "Zimmer Man" ha un carattere eminentemente biografico.

11. Baroncelli Eugenio, Risvolti svelti. Breviario amoroso di vite altrui capitolate, Palermo, Sellerio, 2017.

12. De Stefano Cristina, Scandalose. Vite di donne libere, Milano, Rizzoli, 2017.

13. ACCatino Alfredo, Outsiders: storie di artisti geniali che non troverete nei manuali di storia dell'arte, Milano, Giunti, 2017.

14. Buonanno Errico, Vite straordinarie di nomini volanti, Palermo, Sellerio, 2018.

15. Codignola Matteo, Vite brevi di tennisti eminenti, Milano, Adelphi, 2018.

16. Petrignani Sandra, La corsara. Ritratto di Natalia Ginzburg, Milano, Neri Pozza, 2017; Santagata Marco, Boccaccio indiscreto. Il mito di Fiammetta, Bologna, Il Mulino, 2019. 
Ognuna di queste biografie è sui generis o quasi, e rappresenta un pezzetto della variegatissima terra della prosa - per usare un'espressione di Andrea Cortellessa - del xxi secolo. Tuttavia, la varietà di intenzioni poetiche e di forme biografiche che i testi citati mostrano non può essere trascurata. Per orientarci in un campo così eterogeneo conviene innanzitutto distinguere $\mathrm{i}$ récit lunghi e che si concentrano su un solo personaggio da quelli brevi e serializzati, che si concentrano quindi su più personaggi. Inoltre, bisogna distinguere le biografie propriamente dette, quelle raccontate da un narratore terzo, da quelle scritte in maniera (falsamente) autobiografica, cioè quelle in cui il narratore è lo stesso personaggio biografato, ovviamente diverso dall'autore ${ }^{17}$. Infine, bisogna riconoscere e isolare il carattere narrativo, letterario e finzionale di una biografia. Come ricorda $\operatorname{Cohn}^{18}$ questi concetti non sono equivalenti, e il fatto che il linguaggio della critica letteraria spesso li usi in maniera interscambiabile contribuisce a creare confusione. Ogni biografia intesa come "racconto di una vita" è narrativa ${ }^{19}$, ma non è necessariamente letteraria - non userà cioè sempre gli strumenti linguistici e stilistici della letteratura $\mathrm{a}^{20}$ - né, a maggior ragione, è sempre finzionale - non userà cioè l'immaginazione e l'invenzione in maniera propria e significativa ${ }^{21}$. Ogni biografia contemporanea è

17. Per esempio, come in La morte di Virgilio (1945) di Hermann Broch e nelle Memorie di Adriano (1951) di Yourcenar. In ambito italiano questa modalità enunciativa è stata scelta da Andrea Tarabbia per Il giardino delle mosche. Vita di Andrej Cikatilo (2015).

18. CoHn Dorrit, The Distinction of Fiction, London, The Johns Hopkins University Press, 1999.

19. Per questo è necessario distinguere le biografie vere e proprie dai ritratti o dalle note e voci biografiche, in cui è il carattere descrittivo e informativo ad essere preponderante. Dato che ogni biofiction, come ogni racconto finzionale, è un discorso principalmente narrativo, il narratore ha un'importanza cruciale: è l'enunciazione - variabile e spesso ambigua - del narratore che ci racconta una vita, che ci dà le informazioni sulla storia e sul carattere del biografato, e ci dà accesso alla sua interiorità. Più precisamente, è attraverso gli enunciati del narratore che possiamo guardare da diversi punti di vista e quindi che ci è possibile comprendere e partecipare al suo destino. Per questo motivo a mio avviso sono i differenti tipi di narratore che determinano le differenti tipologie di biografie finzionali.

20. E non, per esempio, quelli del saggio o del pezzo giornalistico.

21. Per fiction intendiamo una "representation portraying an imaginary/invented universe or world" (SCHAEFFER Jean-Marie, "Fictional vs. Factual Narration", in HüHN Peter, Meister Jan Christoph, Pier John, Schmid Wolf [eds.], Handbook of Narratology, vol. I, Berlin-Boston, De Gruyter, 2009, p. 181), che dunque produce un "literary nonreferential narrative text" (COHN Dorrit, The Distinction of Fiction, cit., p. 1). Da un punto di vista testuale, in una biografia, la fiction può manifestarsi in maniera "debole", immaginando o supponendo quello che il biografato potrebbe aver fatto o pensato, o in maniera "forte", entrando direttamente nella sua interiorità. 
invece il risultato della combinazione e dalla gradazione di questi tre elementi: il narrativo, il letterario e il finzionale.

Il prodotto più originale e interessante è quello che combina questi tre elementi dentro un racconto rigorosamente biografico, ovvero il racconto biofinzionale. La grande novità delle scritture biografiche recenti, infatti, risiede in un cambiamento capitale, tanto sostanziale quanto formale (anzi, sostanziale perché formale), avvenuto nel corso del xx secolo, spesso sottovalutato o minimizzato: l'ibridazione tra il discorso fattuale e il racconto finzionale. Concepito come coesistenza di due modalità discorsive opposte ma anche come uno scambio reciproco di strategie $\mathrm{e}$ di retoriche testuali, questo carattere rappresenta un apriori teorico e il punto di partenza pratico del nostro discorso: ovvero l'unico modo per discutere in maniera rigorosa di biofiction, cioè di biografia finzionale. L'importanza che le scritture ibride hanno acquisito nella letteratura occidentale contemporanea è ormai assodata, al punto che sembrano rappresentare l'unica tendenza originale e chiaramente riconoscibile nella produzione narrativa del nostro extrême-contemporain. Nel corso degli ultimi decenni le forme narrative e letterarie che spostano la frontiera fra la fiction e la non-fiction costituiscono, in maniera sempre più evidente, una nuova sfida alla comprensione del nostro contemporaneo. La scrittura fattuale maggiormente sottomessa alle modifiche e alle sovversioni dell'immaginazione nel corso degli ultimi centoventi anni è la biografia, che sembra investire in un modo o nell'altro tutti gli scritti ibridi contemporanei. Con "biofiction" intendo dunque una pratica narrativa che ibrida due generi di natura discorsiva diversa: la biografia e il racconto di finzione. Il termine "biofiction" suggerisce che anche la biografia tradizionale di un personaggio celebre scritta da un terzo può essere innervata dalla fiction e dalle sue leggi. Quando questo avviene, la ricostruzione biografica, anche se è fatta attraverso documenti precisi, non sfugge all'immaginazione di chi la racconta e in definitiva la riscrive. La frontiera di cui parliamo non si posiziona dunque fra due arti (come la letteratura e la fotografia), né fra due generi (come il romanzo e il saggio), ma tra due modi della rappresentazione opposti come la fiction e la non-fiction. In questo senso, un oggetto ibrido non è qualcosa di genericamente composito (che cioè mischia elementi eterogenei) ma qualcosa che contiene elementi appartenenti a campi testuali antinomici.

Per questo, da un punto di vista formale perché ci sia "biofiction" è necessario che i due termini dell'ibridazione, la biografia e il racconto di finzione, siano entrambi chiaramente riconoscibili e che quindi il testo proponga o una finzionalizzazione della biografia di una persona reale o una "biografizzazione" di un personaggio di finzione: solo in questo modo il testo acquisisce il carattere di ibridità, quando è allo stesso tempo vera biografia e vera finzione. L'altra 
condizione è che questi testi mostrino un'intenzione specifica e ambiziosa rispetto al racconto della vita scelta, un'ambizione che non potrebbero perseguire senza ricorrere alla fiction, cioè all'invenzione.

Dato che il campo letterario odierno è proficuamente e irreversibilmente contaminato dalla presenza di fatti realmente accaduti e dalla loro immediata riscrittura, da un punto di vista storico-estetico la biofiction rappresenta la versione iper-contemporanea del genere antichissimo della biografia. All'estero, soprattutto in Francia ${ }^{22}$, questo genere è studiato da una ventina d'anni, mentre in Italia è solo di recente che ha trovato l'attenzione della critica accademica ${ }^{23}$, con un saggio di Riccardo Castellana ${ }^{24}$ e una tesi di dottorato del sottoscritto ${ }^{25}$.

La biofiction rappresenta quindi una tendenza transnazionale: da un punto di vista storico ha nelle Brief Lives (1669-1696) dell'inglese John Aubrey uno dei testi precursori più significativi, nelle Vies imaginaires (1896) di Marcel Schwob un testo cardine - un perno imprescindibile che marca un prima e un dopo nella storia del genere biografico -, e nella Historia Universal de la Infamia (1935) di Jorge Luis Borges un testo-ponte che lo traghetta verso l'età contemporanea. Tutti questi testi dimostrano che l'immaginazione è l'ingrediente necessario per ogni biografia che si vuole diversa da quella positivista, basata esclusivamente sul rigore fattuale. Concepite e utilizzate nelle maniere più disparate, l'immaginazione e l'invenzione della biofiction contemporanea rispondono a un obiettivo comune: decostruire e ricostruire un'identità con l'ambizione di riuscire a dirne di più, e meglio. Vediamo come questo avviene in ambito italiano.

La prima opera autenticamente biofinzionale scritta in italiano ${ }^{26}$ è La sinagoga degli iconoclasti di Rodolfo J. Wilcock, 1972, scrittore argentino di nascita ma che

22. Attraverso gli articoli e i saggi che, a partire dagli anni Duemila, Dominique Viart e Alexandre Gefen hanno dedicato al tema.

23. Il lavoro di Alessandro Iovinelli è incentrato soprattutto sulle manifestazioni autobiografiche dell'autore nel testo, mentre le ricerche di Lucia Boldrini e soprattutto di Valentina Vannucci si concentrano sulla produzione anglosassone e in particolar modo sulle forme di "historiographical metafiction", più che sulle biografie in senso stretto.

24. Castellana Riccardo, "La biofiction. Teoria, storia, problemi”, Allegoria, n.71- 72, 2015, p. 67-97.

25. Mongelli Marco, Narrer une vie, dire la vérité: la biofiction contemporaine, tesi dottorale, Alma Mater Studiorum Università di Bologna. Dottorato di ricerca in Studi letterari e culturali, 30 Ciclo.

26. Durante la prima vague biografica di inizio Novecento, mentre in Francia e in Inghilterra si sperimentavano forme di "new biography", in Italia, sulla scia della ricchissima tradizione delle "vite illustri", D’Annunzio pubblicava nel 1913 una Vita di Cola di Rienzo (all'interno di un progetto che doveva riguardare le vite di uomini 
in Italia ha vissuto a lungo e in italiano ha scritto la parte più significativa della sua opera. La Sinagoga trova la sua collocazione direttamente nella linea AubreySchwob-Borges. Si tratta infatti di una raccolta di trentacinque biografie di personaggi estremamente bizzarri: "inventori deliranti, avventurieri, scienziati e artisti" 27 , secondo Bolaño, o nelle parole di Pasolini "teorici e utopisti spaventosi, totalmente assurdi, dei geni da rinchiudere in manicomio", di cui l'improbabile esistenza è certificata da una nota bibliografica anch'essa indecifrabile.

Lo spazio della biofiction contemporanea si divide quindi in due grandi province, che sono due modalità narrative opposte: quella delle vite brevi che si serializzano e si raccolgono in una macrostruttura e quella della biografia lunga di tipo genericamente romanzesco. Riferendoci al contesto italiano, per quanto riguarda la forma breve, dopo Wilcock, registriamo i racconti su personaggi storici e reali (che riattivano, in chiave moderna, il genere della Vita) come quelli di Dino Baldi ${ }^{28}$ e Eugenio Baroncellii ${ }^{29}$ e quelli che, proprio seguendo la lezione di Wilcock, costruiscono una mini enciclopedia di personaggi inventati e più o

illustri ma anche oscuri), che è di fatto una riscrittura di una cronaca medievale ma in cui è possibile scorgere un interesse molto moderno per la singolarità dell'essere umano. Paradossalmente più attento al possibile incontro fra resoconto documentato e romanzo è Benedetto Croce, che proprio perché deplora il genere a lui contemporaneo della biografia romanzata, pubblica nel 1936 sei Vite di avventure, di fede e di passione, in cui vuole mostrare che è possibile coniugare acribia storica e vivacità del racconto. Nel 1939, con il Lucrezia Borgia di Maria Bellonci fa l'esordio sulla scena italiana il romanzo biografico di ispirazione psicologica o psicanalitica che molta fortuna, sebbene non priva di polemica, stava avendo in altri paesi europei. Con Narrate, nomini, la vostra storia (1942), Alberto Savinio raccoglie i ritratti biografici, apparsi nel decennio precedente su riviste e giornali, dedicati a personaggi antichi e moderni, dando vita a quella che è la prima raccolta di biografie in Italia nel Novecento. Infine, nel 1947, Anna Banti con Artemisia compie un'operazione biografica originale a partire da un personaggio realmente esistito, la pittrice secentesca Artemisia Gentileschi. Nessuna di queste opere partecipa però dell'ethos tutto contemporaneo della biofiction. Nemmeno le opere a metà tra il racconto di vita e la critica letterarie scritte a partire dagli anni ' 70 da giornalisti come Lajolo (su Fenoglio e Pavese), da critici letterari come Citati, Siciliano e Cattaneo (e oggi come Santagata) e da scrittori come Mario Tobino e Natalia Ginzburg possono dirsi vicini al nostro genere. Nessuna di esse, infatti, possiede allo stesso tempo un impianto saldamente biografico e un uso moderno e audace della fiction letteraria.

27. Bolaño Roberto, "Gli inventori deliranti", in Traparentesi, trad. it. Maria Nicola, Milano, Adelphi, 2009, pp. 292-294.

28. BALDi Dino, Morti favolose degli antichi, Macerata, Quodlibet, 2010; ID., Vite efferate di papi, Macerata, Quodlibet, 2012.

29. Baronceldi Eugenio, Libro di candele. 267 vite in due o tre pose, Palermo, Sellerio, 2008; ID., Mosche d'inverno. 271 morti in due o tre pose, Palermo, Sellerio, 2010; ID., Falene. 237 vite quasi perfette, Palermo, Sellerio, 2012. 
meno fantastici, come Ermanno Cavazzoni ${ }^{30}$ e Giuseppe Pontiggia ${ }^{31}$, e spingendoci nella contemporaneità e vedendo aumentare quindi il grado di ibridazione, come i Personaggi precari (2007) di Vanni Santoni e le biografie letteralmente indecidibili di Davide Orecchio (Città distrutte, 2012).

Per quanto riguarda la forma lunga invece dobbiamo innanzitutto escludere dal campo biofinzionale - oltre alle moltissime biografie o "romanzi biografici" di largo consumo ma di scarsa consapevolezza - anche i generi finzionali alla prima persona, come l'autobiografia di Lucrezio o il diario segreto di Cesare immaginate da Luca Canali (1994 e 1995): sulla scia di Yourcenar e Broch queste opere mimano infatti un discorso non biografico ma autobiografico, in cui cioè è il personaggio biografato a parlare direttamente.

Possiamo quindi considerare e distinguere due macro-categorie: a) una costituita dai lavori biografici intorno a un personaggio storicamente attestato da parte di romanzieri: molto diverse fra loro queste opere possono essere però ordinate secondo un grado via via crescente di ibridazione finzionale: passiamo quindi da romanzi biografici più o meno tradizionali come quelli di Affinati (L'uomo del futuro, 2016), Arpaia (L'angelo della storia, 2001) o Nove (Tutta la luce del mondo. Il romanzo di San Francesco, 2014), a opere più complesse come quelle di Camilleri (Il re di Girgenti, 2001), fino a faction che incrociano biografia, riflessione storica e narrazioni in modo quasi inestricabile: penso in particolare a Genna (Hitler, 2008), Wu Ming (Timira, 2012), e a tutta l'opera di Edgardo Franzosini e Andrea Tarabbia; b) una seconda che chiamerei meta-biografia, in cui cioè la ricerca biografica è o mimata all'interno della diegesi (e solo indirettamente informa l'opera nel suo complesso), come in Lo stadio di Wimbledon (1983) di Daniele Del Giudice, o, in maniera minore in La notte della cometa (1984) di Sebastiano Vassalli, oppure è rappresentata obliquamente all'interno di un pastiche come Tutto il Ferro della Tour Eiffel (2002) di Mari, o da un narratore-testimone come in Io venia pien d'angoscia a rimirarti (1990), sempre di Mari.

In generale, potremmo suddividere la produzione biofionzionale italiana contemporanea in tre grandi categorie, in tre idealtipi: il racconto breve e serializzato, il faction di inchiesta storico e quello che chiamerei "il nuovo romanzo biografico". Ciascuno di questi idealtipi è un'evoluzione di forme intermedie tra il discorso finzionale e quello fattuale come la biografia romanzata o il saggio storico e rappresenta una delle tendenze più importanti della biofiction italiana,

30. Cavazzoni Ermanno, Vite brevi di idioti, Milano, Feltrinelli, 1994; ID., Gli eremiti del deserto, Macerata, Quodlibet, 2016.

31. Pontiggia Giuseppe, Vite di uomini non illustri, Milano, Mondadori, 1993. 
in particolare per quanto riguarda i diversi usi dell'invenzione e dei documenti storici e quindi le sue possibili ambizioni testimoniali. In questo modo la biofiction mostra di essere una pratica discorsiva particolarmente vivace e varia, che facendo reagire il récit de vie con la Storia e con la finzione mostra la sua centralità nell'estetica contemporanea.

I tre rappresentanti italiani più importanti di queste modalità diverse di biofiction sono rispettivamente Davide Orecchio, Andrea Tarabbia e Edgardo Franzosini.

Edgardo Franzosini ha pubblicato finora cinque testi a carattere biografico: Bela Lugosi. Biografia di una metamorfosi, (1984, poi 1998), Il mangiatore di carta, (1989, poi 2017), Raymond Isidore e la sua cattedrale, (1995), Sotto il nome del cardinale, (2013) e Questa vita tuttavia mi pesa molto (2015) ${ }^{32}$. Sono opere diverse fra loro per impianto narrativo (che oscilla dalla referenzialità storica quasi perfetta di Sotto il nome del cardinale all'incedere perlopiù romanzesco di Questa vita tuttavia mi pesa molto), ma non per intenzione poetica: restituire la vita di personaggi realmente esistiti, eccentrici e molto letterari, da Bela Lugosi a Bugatti, appunto, attraverso un'istanza narratoriale molto presente, che assume sempre le sembianze del biografo, e che a partire dai quei codici enunciativi racconta la vita dei suoi personaggi.

Andrea Tarabbia mi sembra invece il rappresentante migliore di quel faction storico che è la forma più comune e più celebre di scrittura ibrida contemporanea. Seguendo il solco tracciato dal Limonov di Carrère, Tarabbia racconta le vite di due efferati criminali realmente esistiti ma trasfigurati nella finzione biografica: Marat Bazarev in Il Demone a Beslan (2011) e Andrej Čikatilo in Il giardino delle mosche $(2015)^{33}$. In questi testi il narratore si ibrida in maniera più significativa rispetto a quelli di Franzosini, cambia nel corso del testo e ci mostra le vicende da molti punti di vista, quelli del carnefice in primis, senza mai coincidere con l'autore. L'istanza biografica è infatti diffusa nel testo ed emerge localmente in momenti significativi del récit della vita.

Lo scrittore a mio avviso più importante del panorama biofinzionale contemporaneo (italiano e non solo) è Davide Orecchio. Il suo esordio, la raccolta di racconti Città distrutte. Sei biografie infedeli (2012), si pone all'incrocio di molte vie - il saggio storico e il récit de vie, la precisione archivistica e il pathos romanzesco, la speculazione filosofica e il lirismo - che la biografia struttura in maniera coerente e unitaria. I racconti raccontano la vita di personaggi realmente esistiti,

32. Rimbaud e la vedova, pubblicato nel 2018, si concentra solo su un momento della vita del biografato.

33. Anche Carlo Gesualdo, il compositore cinquecentesco protagonista del recentissimo Madrigale senza suono (2019) appartiene alla medesima categoria. 
celebri o meno, ma lo fanno in maniera "infedele" nel senso che esse riferiscono i dati biografici e i documenti di archivio per manipolarli, falsificarli e trasfigurarli nell'immaginazione letteraria. Attraverso un'istanza narratoriale molto ambigua e l'uso rischioso ma consapevole dei documenti storici e della creazione letteraria, Città distrutte riesce a produrre una riflessione sulla storia recente, italiana ma non solo. Testimoniando e documentando le storie private dentro la Storia collettiva, riesce a dire una verità non banale e più profonda su quegli uomini e donne particolari di cui racconta la vita e si rivela un testo complesso e ambizioso, allo stesso tempo innovativo sul piano della forma e dell'intenzione etica ed estetica.

In conclusione: l'obiettivo della biofiction contemporanea è quello di ricostruire la vita e l'immagine di una persona su una base non solamente documentaria. Questa immagine può fondarsi su dettagli e aneddoti bizzarri, su comportamenti e caratteristiche ritenuti rivelatori e sui quali le proporre un ritratto vivo, certo letterario ma in ogni caso fondato storicamente. Questo genere vuole insomma dire una verità che non è semplicemente letteraria, poetica, ma veramente ibrida, insieme complessa e immediata. Per farlo, utilizza la fiction non come una menzogna mascherata ma come uno strumento euristico che ci fa comprendere qualcosa che non potremmo conoscere altrimenti.

Marco Mongelli Università di Bologna, Université Sorbonne Nouvelle - Paris 3 
